\title{
Review
}

\section{Genetic Approach to Elucidation of Sasang Constitutional Medicine}

\author{
Bu-Yeo Kim, Seongwon Cha, Hee-Jeong Jin and Sangkyun Jeong \\ Division of Constitutional Medicine Research, Korea Institute of Oriental Medicine, 461-24, Jeonmin-dong, \\ Yuseong-gu, Daejeon, 305-811, Korea
}

\begin{abstract}
Sasang Constitutional Medicine (SCM) offers a medical principle that classifies humans into four constitution groups and guides their treatment with constitution-matched medical assistance. The principle of this traditional medicine, although requires significant scientific support, appears to suggest a genetic influence on constitution type. The relative frequency of constitution types in a population, for instance, has remained relatively constant since Jema Lee first described them from his observations. In addition, the body compartment concept of SCM appears to be related to the anterio-posterior patterning of the embryonic gut and associated internal organs. This study describes the attributes of the constitution concept of SCM that can be interpreted in the language of genetics and current approaches to identity the genetic factors that make up the constitution. These efforts should make it possible to interpret the principle of this traditional medicine scientifically. Considering the recent trend in medicine that pursues individualized or tailored medical offerings, once SCM is proven to be explainable with scientific evidence, it will be able to contribute to and take a place in the rapidly evolving medicine environment.
\end{abstract}

Keywords: constitution - polymorphism - tailored medicine - traditional medicine

\section{Introduction}

Since the discovery of antibiotics, medicine has achieved considerable success in understanding and providing cures for diseases. Knowledge from related research fields including molecular biology, physiology and genetics has contributed to the advance of medicine. As the field of medicine developed, Archibald Garrod perceived that individual differences inherently engraved would be medically significant and should be considered during treatment (1). With this observation as a turning point, medical science has been moving progressively from solely focusing on disease toward the consideration of the individuality of

All authors contributed equally to this work.

For reprints and all correspondence: Sangkyun Jeong, $\mathrm{PhD}$,

Department of Medical Research, Korea Institute of Oriental Medicine

(KIOM), 461-24 Jeonmin-dong, Yuseong-gu, Daejeon 305-811,

Republic of Korea. Tel: +82-42-868-9314; Fax: +82-42-868-9480;

E-mail: skjeong@kiom.re.kr the patient. This trend has accelerated with recent advances in science, especially the completion of the Human Genome Project and the International HapMap Project, allowing the potential for personalized medicine $(2,3)$. Thus, individualities such as differences in susceptibilities to diseases or medications are being sought and verified at the level of genetic variations (4). With the success of these efforts, human beings are expected to benefit from more effective prevention and treatment of diseases through such remedies as tailored applications of drugs. A challenge arising from increasingly individualized courses of medical treatment is the great amount of patient information that must be generated.

One alternative to such purely individual medical approaches would be a typological approach in which individuals are categorized into certain groups defined by similarities in their physiology, psychological characteristics, behavioral properties or body constitution through scientifically standardized methods. Indeed, from 
the earliest ages, medical practice has been performed on a typological basis in both the West and the East. Hippocrates' demonstration of the four humors and its succession to Galenus to develop humoral pathology are examples of the old western tradition. More recently, Kretschmer categorized humans into two groups, schizothymic and cyclothymic, based on their psychological characteristics (5). A similar tradition is also found in the East. Based on traditional Chinese medicine (TCM), Wang classifies humans into seven constitution groups based on physiological and physical status (6). Wang stresses the importance of precise diagnosis of the constitution, as it affects the occurrence, development and prognosis of disease and is the basis for prescribing herbs. Interestingly, Wang suggests that the constitution can change according to one's current disease. In Ayurveda, Indian constitutional medicine, humans are classified into three Doshas: Vata, Pitta and Kapha, irrespective of race, language, ethnicity and religion $(7,8)$. In Ayurvedic medical theory, the constitution of each individual is determined according to which dosha is dominant as he/she expresses various physical, physiological and psychological characteristics that are attributed to each dosha (9). Unlike in Chinese tradition, the Ayurvedic constitution is constant throughout one's lifetime, suggesting genetic involvement in its formation.

Sasang Constitutional Medicine (SCM) was established by Jema Lee under the Korean medical tradition (10-13). It classifies humans into four constitution types, Taeyangyin (TY Type), Tae-eumin (TE Type), Soyangyin (SY Type) and Soeumin (SE Type) depending on the nature of an individual's physiological, psychological and physical characteristics that lead to differential responses to herbs (10). On the basis of these characteristics, the constitution of each individual can be determined, and patients can thus be provided with constitution-matched medical assistance. As is the case for the Ayurvedic constitution, one's constitution in SCM is unchanging and determined by inherent characteristics. This study will discuss the principles of SCM in biological and genetic terms and will address current efforts to discover scientific evidence for the mechanisms of this traditional medicine.

\section{Biological Interpretation of Sasang Physiological Concept}

\section{Constitutional Classification in SCM}

According to Jema Lee's Donguisusebowon (Longevity and Life Preservation in Oriental Medicine) (11), the state of balance among the physiological functions that are defined by four representative internal organs is the most important factor for determining the SCM constitution type (Table 1). Specifically, the lung, spleen, liver and kidney are the main organs that represent the respiratory, digestive, preservative and excretive functions, respectively. The names of these organs symbolize physiological function rather than anatomical identity. The lung and liver are paired to determine the physiological balance between the usage and the storage of energy, respectively, while the spleen and kidney are responsible for balancing the digestive and excretory functions. Based on the nature of the balances between the paired organs, every person is born with one of the four constitutions. The TY type is characterized by the dominance of the lung function over the liver function, whereas the TE type features an opposing dominance. A reciprocal relationship is also found between the SY and SE types based on whether the spleen or kidney function is superior, respectively.

\section{Biological Relevance of SCM}

A challenge for those who would more closely integrate SCM with modern medical science is the anatomical definition of the four internal organs of SCM. Considering that the four internal organs in SCM are believed to be defined according to the functional unit, these four organs should not be regarded as simple counterparts of modern anatomy. Currently, the lung, through which Qi flows, is thought to be SCM's functional name for the modern anatomical respiratory organs, including the lungs, bronchi and skin. The liver, which might include the liver, gall bladder and small intestine, is thought to represent the organs involved in energy absorption. The spleen, which controls food intake, would designate the pancreas, spleen and the stomach in modern terminology.

Table 1. Characteristics of the four types of Sasang constitution ${ }^{\mathrm{a}}$

\begin{tabular}{lllll}
\hline Feature & TY & TE & SY & SE \\
\hline Physique & Big & Big & Small & Small \\
& Slender waist & Thick waist & Developed chest & Weak chest \\
Small hip & Developed hip \\
Physiology & Respiratory $>$ Preservative & Respiratory $<$ Preservative & Digestive $>$ Excretive & Digestive $<$ Excretive \\
Psychology & Heroic & Cautious & Hot tempered & Nervous \\
& Creative & Pragmatic & Easily bored & Logical \\
& Progressive & Reflective & Lively & Neat \\
\hline
\end{tabular}

${ }^{a}$ This table was constructed by modifying two previously reported tables $(10,39)$.

${ }^{\mathrm{b}}$ The physiological features of each type are represented by the state of functional balance using an inequality mark. 
The kidney can be thought to represent the functional group containing the kidneys, bladder and large intestine, which are responsible for excretion. Therefore, the four organs defined in SCM are actually related to nearly all of the internal organs described in modern medicine.

As will be discussed in the following section, the engagement of the four internal organs into the whole of the human physiological system could be explained by postulating that pleiotropic factors may play roles in the early developmental stage to differentiate the balances of the internal organs among the four constitution types. Although the inter-relationships between the internal organs of SCM and human physiology would be difficult to determine, some physical characteristics common to particular types of the four constitutions could be confirmed relatively easily. For example, the TY type is thought to develop the nape of the neck, the SY type to develop the chest, the TE type to develop the waist and the SE type to develop the hip (Table 1). In addition to these physical differences, psychological characteristics also show a differential distribution among the four constitution types (Table 1). For example, the SY type has a more extroverted character than the TE and SE types, while the SE type displays a more judging characteristic as a psychological feature compared to the SY and TE types based on the Meyers-Briggs Type Indicator (MBTI) (14). These phenomena imply a functional linkage of the organs with other parts of the body. Based on these different physical and psychological traits among the four types of constitution, practitioners of SCM can determine the constitutions of individual patients. However, because the diagnosis of constitution is determined by subjective judgments of individual practitioners of SCM, establishing objective criteria for a diagnosis of the constitution is of high priority in SCM.
Specifically, assessment of responses to specific drugs is one of the major determinants of constitution in SCM and may represent biochemical differences in drug metabolism among constitution types $(15,16)$. Furthermore, according to SCM, every person is born with one of four types of constitution, which implies the involvement of genetic heredity in the constitution and the possible presence of molecular factors determining the constitution.

\section{Genetic Concepts in the Principle of SCM}

The logical structure of Confucianism that recognizes objects as quaternary structures was widely adapted in the establishment of SCM's medical principle (10). Assigning individuals into one of four constitution types based on the nature of the physiological balances among the four representative organs is a typical example. This logical structure is further utilized to describe the human body in terms of its structural architecture. According to Donguisusebowon, three boundaries divide the human body into four compartments along the vertical axis; from top to bottom, these four compartments are termed the upper-most, upper-middle, lower-middle and lower-most burn-centers (UBC, UMBC, LMBC and LBC for short) (11). The four body compartments of UBC, UMBC, LMBC and LBC are described by Jema Lee as being in respective functional relationships to the lung, spleen, liver and kidney groups, respectively. Although this compartmentalization concept does not appear to reflect the anatomical characteristics of the adult body, it is well correlated with the architectural property of the developing fetal gastro-intestinal tract (Fig. 1). The esophagus, which belongs to UBC, provides a place where the laryngotracheal groove develops to give

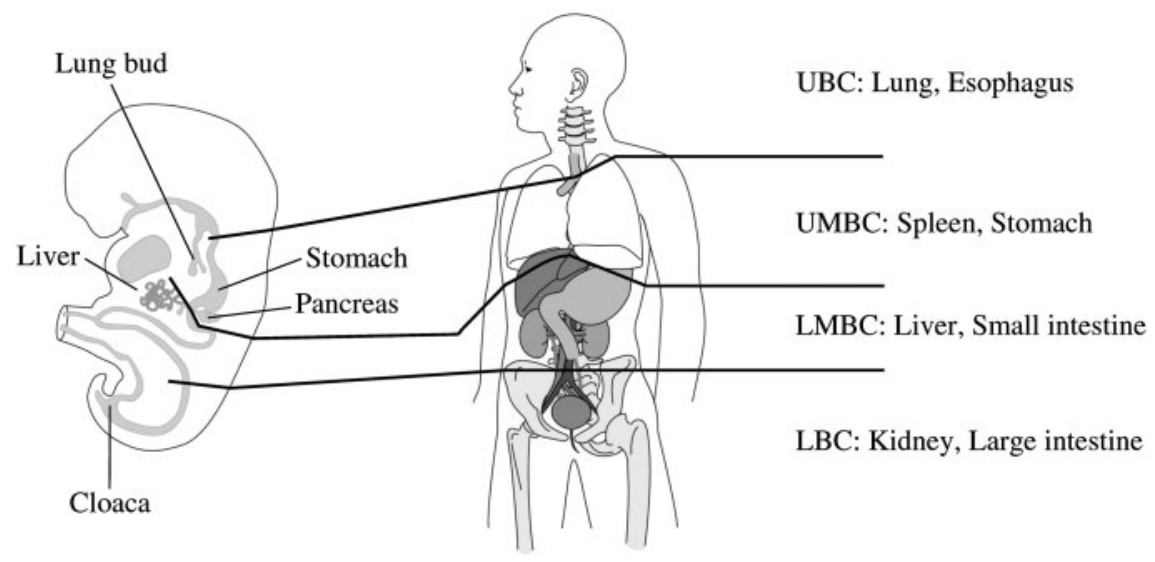

Figure 1. Correlation of four burn-centers to their respective organs. In SCM's physiology, each of the four burn-centers, UBC, UMBC, LMBC and LBC, has been postulated to correspond to the representative body domain that is grossly defined by the area constituting the cervical, thoracic, lumbar or sacral vertebrae, respectively. These body domains are then correlated to four representative organs (lung, liver, spleen and kidney). This does not appear to be in agreement with the anatomical characteristics of the adult body but does appear to agree with that of a developing fetus. The boundaries that demarcate each burn-center in a developing fetus and an adult are designated with black lines. The organ names that correspond to each burn-center are followed by the names of the burn-centers. 
rise to the lungs, the representative UBC organ (17). UMBC encompasses the developing gut area where the putative stomach evolves. LMBC covers the area of the gut that will form the small intestines. Interestingly, the liver is connected with the fetal gut just below the stomach through the bile duct, providing logical consistency that the liver and small intestine belong to LMBC (18). The kidneys develop from the mesenchymal cells of invading uretric buds, the protrusions of mesonephric ducts which connect the metanephros, the future kidney and cloaca at the end of the hindgut, justifying the assignment of the kidney and large intestine to LBC (19).

The constitution in SCM thus appears to relate the body domains defined by the development pattern of internal organs, particularly relating the development patterns in the gut to the representative physiological functions defined by SCM's four internal organs. This body compartmentalization is reminiscent of the anterio-posterior (AP) axis patterning that can be observed during animal development. In drosophila development, the AP identity is determined by the precise regulation of a subset of genes, including homeobox-containing genes (20). Similarly, AP patterning is also elaborated by homologous hox genes in mammalian development (21). In this regard, the body compartment concept used in SCM, which obviously originates from oriental medical insights, may have a genetic basis, as does the axial patterning of animal development.

Jema Lee also implied that the frequencies of each constitution type in a population remain constant over generations, which is a principle that is well known in genetics as the Hardy-Weinberg equilibrium. In line with this, Jema Lee insisted that the constitution type one carries is an inborn feature. It is interesting that these statements were made by a man without a background in genetics, emphasizing that the insights acquired on the basis of traditional oriental medicine may have suggested a principle that modern science has now proven.

\section{From Physiology to Constitution Genes}

Physiological variations that are observed between different species or within the same species are derived from genetic variations. Wide ranges of phenotypic variations with respect to behavioral characteristics, physiological properties and susceptibilities to diseases were also found in different mouse strains that were derived from a few wild-mouse ancestors through systematic breeding in laboratory environments (22). These mice share the same genetic contents in terms of the type, number and ordered structure of the genes on their genomes. Therefore, the observed variations are largely attributed to small sequence differences in the genome that differentiate the structural, functional or quantitative properties of gene products. The outcome of genetic variation will give rise to differences in the protein structure or in the patterns of expression regulations. The latter case has recently gained significant attention from researchers with the development of procedures allowing the precise quantification of gene expressions. For example, Yan et al. (23) reported that variation of the regulatory region of the $A P C$ gene leading to a low level of gene expression is correlated with susceptibility to colon cancer. The physiological differences among constitution types would be similarly manifested as a result of variations of the regulatory regions of genes, leading to differentiation in the expression regulation.

Individuals of the same constitution type are considered to share some common features that are defined by body constitution, physiology, or psychology. For example, individuals of the TE type are said to share the following characteristics: a relatively high perspiration rate, high propensity to become obese due to stronger liver function compared to lung function, persistence and an introverted character. No thorough scientific effort to extract common features from among these properties appears to have been conducted. As it is recognized that the constitution in SCM is genetically determined, it is necessary to address how genetics would operate to bring the features characterizing those constitutions together. This rationalizing procedure will shed light on the role of potential constitution genes. As stated in the previous section, axial patterning in the early developmental stage appears to be in close relation to the determination of constitution. The hox genes responsible for this early developmental process are in turn regulated by a subset of genes known as gap genes and segmentation genes in drosophila. The regulatory flow of gene expressions is initiated by the activation of maternal effect genes in the oocyte upon fertilization. Evidence has confirmed the conservation of such regulatory networks in animal development (21). Given that genes in this category exert their effects on various lineages of cells simultaneously, the variations on these genes would give rise to multiple phenotypic variations including body constitution, behaviors, physiology and psychology. In this regard, these genes are most likely to manifest the properties of constitution-associated genes. We are, therefore, currently focusing on these genes to determine whether the variations in these gene regions are associated with specific constitution types.

\section{Seeking the Genetic Elements Responsible for Constitution}

\section{Characterization of Constitution as an Initial Step of Genetic Study}

To translate traditional constitutional medicine into the language of modern genetics, an objective and reliable characterization of constitution types will be 
very important. A similar problem would be faced in selecting the case and control in human population studies of complex diseases. This initial step in the population study will strongly determine the success in finding disease-associated genes (24). As such, the exact characterization of constitution types will significantly influence whether constitution-associated genes will be sought.

To meet the criteria of objectivity and reliability often obscured due to the subjective diagnosis of SCM experts, two points should be considered. First, ambiguously characterized subjects should be excluded. Genetic studies of Ayurvedic constitutional medicine have focused on only three extreme constitution types among the seven total types constructed by the relative proportions of the three Doshas (Vata, Pitta and Kapha) $(9,25)$. The exclusion of the intermediate types formed by combinations of the three major constitutions can be a smart strategy to avoid any ambiguity caused by mixed genetic effects that can be manifested in these intermediate types for a genetic analysis of constitution. Second, objective and precise criteria for the classification of subjects should be established. Subjects used in SCM genetic studies have been typed by self-assessment questionnaires (26-32). However, typing constitution with a questionnaire does not appear to be as reliable as the subjective diagnosis of SCM experts, despite the good objectivity of questionnaires $(33,34)$. It would be more appropriate to classify the individual's constitution based on the herbal responses, as each constitution type has different sensitivity to herbal medicine $(10,11)$. For example, a subject of the TE type can be distinguished from other types by favored responsiveness to ma huang (Ephedra sinica; principal constituents are ephedrine and pseudoephedrine), while it may cause significant adverse effects including insomnia and tachycardia in other types $(35,36)$. Ginseng (Panax ginseng) is another example of a drug that is used for the determination of constitution in which the SY type shows adverse effects while the SE type does not (11). However, a classification of constitution based on herbal responsiveness should be performed on a strictly limited basis and with great care owing to the ethical issues involved.

To assure reliability and objectivity in the constitution typing for the genetic analysis of the Sasang constitution, only individuals who had exhibited favorable responses to constitution medicines administered for at least 1 month were included, which simultaneously resulted in the removal of the subjects showing ambiguous herbal responsiveness. A Case Report Form (CRF) for each subject consisting of various anthropometric measurements and questionnaires for personality, general health conditions, responses to herbal medicines and other factors was also collected. The objective measurements in the CRF can be used along with hematological values to interpret the morphological and physiological manifestation of each constitution in genetic studies.

\section{Genetic Analysis of Constitution in SCM}

The Sasang constitution appears similar to complex traits such as obesity, diabetes and hypertension involving multiple genetic factors. However, unlike those complex traits, which are manifested at certain points in a patient's lifetime through the combined effects of genetic and external environmental factors, constitution is inherently determined. This principle is the reason for the current focus on searching for genetic determinants of the Sasang constitution.

As the four constitution types are thought to be related to all aspects of physical, physiological and psychological traits, there is a limit to the single-gene approach to elucidating the genetic basis of SCM constitutions. Earlier genetic studies on SCM searched exclusively for the effects of single candidate genes (26,29-32). They found that the polymorphisms of interleukins including interleukin 1 receptor antagonist, alpha and beta showed susceptibility to obesity in TE females (29-31) and that a Pro/Ala variant in peroxisome proliferator-activated receptor gamma showed 15-fold increased susceptibility to ischemic stroke in TE subjects (32). Although the results from these studies are very encouraging, a more comprehensive and systematic approach will be necessary to elucidate the genetic constituents accounting for constitution in SCM rather than a case-by-case identification of the constitution-associated genes. In related research, two genetic studies have revealed the relationship between highly polymorphic human leukocyte antigens and the constitutions of both TCM and Ayurveda (9,37-39). A recent genomic approach has elucidated the different levels of genome-wide expression and corresponding biochemical parameters according to three contrasting Ayurvedic constitutions (25). Three later genetic studies have made contributions to the integration of traditional and modern medical sciences by determining multi-genetic factors to differentiate physiologic constitutions.

After the completion of the International HapMap Project, genetic individuality has greatly changed the concept of how disease occurs and what treatment strategies should be applied. Moreover, the recent development of SNP microarrays makes it possible to detect genetic variations on a genome-wide level. In this context, we are pursuing comprehensive genomic studies that include whole-genome association, linkage and expression analysis according to unrelated or familial subjects as well as candidate gene approaches to identify constitutionassociated genes selectively. Through these strategies, it is expected that nearly all major genetic determinants of constitution will be identified. In fact, the putative chromosomal region related to the TE type was found 
to have a high logarithm of odd score from a wholegenome scan analysis with familial subjects (40). Recently, it was observed that the effects of risk alleles of fat masses and an obesity-associated gene (FTO) on BMI differed among constitution types, suggesting genetic interactions between constitution genes and FTO (Cha et al., manuscript in submission). Although these studies represent only the beginning of the genetic elucidation of the SCM constitution, the precise characterization of constitution types and their subsequent systematic genetic analyses assure accurate and productive communication between the fields of SCM and genetics.

\section{Conclusions}

The paradigm in medicine is shifting from a focus on disease to a focus on the patient, as the susceptibility and prognosis of a patient to a given disease is recognized to be dependent on individual differences. This trend is further facilitated by improvements of the laboratory environment, such as the development of high-throughput equipment that can be used to correlate phenotypic traits with specific genetic variations. Recently, emerging biological systems focusing on integrating all types of complex biological information in the simulation of living organisms will provide methods to advance research into individuality. Although a biological approach to the delineation of constitution in SCM appears to resemble modern personalized medicine, there is a simple but important difference between these two approaches. While SCM has attempted to elucidate all types of human characteristics into one theoretical concept, modern personalized medicine focuses mainly on the treatment of specific diseases. Therefore, if a more detailed genetic approach to the identification of the four constitution types can be developed, SCM could provide an alternative and unique model of personalized medicine that could be applied in the prevention and treatment of human physiological and psychological diseases. Moreover, the success of this work will allow the philosophical basis of oriental medicine to be rewritten in the language of science.

\section{Acknowledgments}

The authors would like to thank Dr Jong Yeol Kim (Department of Medical Research, Korea Institute of Oriental Medicine) for his assistance with the detailed interpretation of SCM as an oriental medical doctor.

\section{Funding}

Korea Science and Engineering Foundation (KOSEF) grant funded by the Korean Government (MEST) (Grant No M10643020004-08N4302-00400).

\section{References}

1. Childs B. A logic of disease. In: Scriver CR, Beaudet AL, Sly WS, Valle D (eds). The Metabolic and Molecular Bases of Inherited Disease. New York: McGraw-Hill, Inc, 1995, 229-57.

2. Collins FS, Green ED, Guttmacher AE, Guyer MS. A vision for the future of genomics research. Nature 2003;422:835-47.

3. Frazer KA, Ballinger DG, Cox DR, Hinds DA, Stuve LL, Gibbs RA, et al. A second generation human haplotype map of over 3.1 million SNPs. Nature 2007;449:851-61.

4. Roses AD. Pharmacogenetics and the practice of medicine. Nature 2000;405:857-65.

5. Kretschmer E. Physique and Character An Investigation of the Nature of Constitution and of the Theory of Temperament. New York: Harcourt, Brace \& Company, 1925.

6. Wang Q. Theories of Physical Constitutions of Traditional Chinese Medicine. Beijing: Chinese Medical Science and Technology Publishing Company, 1995.

7. Swoboda R. Prakriti: Your Ayurvedic Constitution. Delhi: Motilal Banarasidass Publishers, 1996.

8. Hankey A. Ayurvedic physiology and etiology: Ayurvedo Amritanaam. The doshas and their functioning in terms of contemporary biology and physical chemistry. J Altern Complement Med 2001;7:567-74.

9. Bhushan P, Kalpana J, Arvind C. Classification of human population based on HLA gene polymorphism and the concept of Prakriti in Ayurveda. J Altern Complement Med 2005;11:349-53.

10. Shim EB, Lee S, Kim JY, Earm YE. Physiome and sasang constitutional medicine. J Physiol Sci 2008;58:433-40.

11. Lee J. Longevity \& Life Preservation In Oriental Medicine. Seoul: Kyung Hee University Press, 1996.

12. Leem KH, Park HK. Traditional Korean medicine: now and the future. Neurol Res 2007;29(Suppl 1):S3-4.

13. Cha WS, Oh JH, Park HJ, Ahn SW, Hong SY, Kim NI. Historical difference between traditional Korean medicine and traditional Chinese medicine. Neurol Res 2007;29(Suppl 1):S5-9.

14. Chae H, Lyoo IK, Lee SJ, Cho S, Bae H, Hong M, et al. An alternative way to individualized medicine: psychological and physical traits of Sasang typology. J Altern Complement Med 2003;9:519-28.

15. Choi JS, Jung SW, Ju JC, Lee SW, Kim KY, Kim HM. Cytokine production regulation in human astrocytes by a herbal combination (Yuldahansotang). Immunopharmacol Immunotoxicol 2002;24:55-67.

16. Jeong HJ, Lee HJ, Hong SH, Kim HM, Um JY. Inhibitory effect of Yangkyuk-Sanhwa-Tang on inflammatory cytokine production in peripheral blood mononuclear cells from the cerebral infarction patients. Int J Neurosci 2007;117:525-37.

17. Mendelson CR. Role of transcription factors in fetal lung development and surfactant protein gene expression. Annu Rev Physiol 2000;62:875-915.

18. Zhao R, Duncan SA. Embryonic development of the liver. Hepatology 2005;41:956-67.

19. Vize PD, Seufert DW, Carroll TJ, Wallingford JB. Model systems for the study of kidney development: use of the pronephros in the analysis of organ induction and patterning. Dev Biol 1997;188: 189-204.

20. Scott MP, Carroll SB. The segmentation and homeotic gene network in early Drosophila development. Cell 1987;51:689-98.

21. Pearson JC, Lemons D, McGinnis W. Modulating Hox gene functions during animal body patterning. Nat Rev Genet 2005;6:893-904.

22. Bogue MA, Grubb SC, Maddatu TP, Bult CJ. Mouse Phenome Database (MPD). Nucleic Acids Res 2007;35:D643-9.

23. Yan H, Dobbie Z, Gruber SB, Markowitz S, Romans K, Giardiello FM, et al. Small changes in expression affect predisposition to tumorigenesis. Nat Genet 2002;30:25-6.

24. Cardon LR, Bell JI. Association study designs for complex diseases. Nat Rev Genet 2001;2:91-9.

25. Prasher B, Negi S, Aggarwal S, Mandal AK, Sethi TP, Deshmukh SR, et al. Whole genome expression and biochemical correlates of extreme constitutional types defined in Ayurveda. $J$ Transl Med 2008;6:48.

26. Um JY, Joo JC, Kim KY, An NH, Lee KM, Kim HM. Angiotensin converting enzyme gene polymorphism and traditional Sasang 
classification in Koreans with cerebral infarction. Hereditas 2003;138:166-71

27. Um JY, Lee JH, Joo JC, Kim KY, Lee EH, Shin T, et al Association between tumor necrosis factor-alpha gene polymorphism and Sasang constitution in cerebral infarction. Am J Chin Med 2005;33:547-57.

28. Um JY, Kim HM, Park HS, Joo JC, Kim KY, Kim YK, et al. Candidate genes of cerebral infarction and traditional classification in Koreans with cerebral infarction. Int J Neurosci 2005; 115:743-56.

29. Lee JH, Kwon YD, Hong SH, Jeong HJ, Kim HM, Um JY Interleukin-1 beta gene polymorphism and traditional constitution in obese women. Int $J$ Neurosci 2008;118:793-805.

30. Um JY, Kim HM, Mun SW, Song YS, Hong SH. Interleukin-1 receptor antagonist gene polymorphism and traditional classification in obese women. Int $J$ Neurosci 2006;116:39-53.

31. Song JS, Jeong HJ, Kim SJ, Son MS, Na HJ, Song YS, et al. Interleukin-1alpha polymorphism $-889 \mathrm{C} / \mathrm{T}$ related to obesity in Korean Taeumin women. Am J Chin Med 2008;36:71-80.

32. Lee BC, Doo HK, Ahn SY, Byun SH, Kim SI, Park HK, et al. Peroxisome proliferator-activated receptor-gamma Pro12Ala polymorphism is associated with the susceptibility to ischemic stroke in Taeeumin classified by Sasang medicine. Neurol Res 2007;29(Suppl 1):S32-7.

33. Kim TY, Yoo JH, Lee EJ, Koh BH, Song IB. The study on the upgrade of QSCC II (I). J of Sasang Const Med 2003;15:27-38.
34. Lee SG, Kwak CK, Lee EJ, Koh BH, Song IB. The study on the upgrade of QSCC II (II). J of Sasang Const Med 2003; 15:39-49.

35. Kim HJ, Kim JY. A clinical report about the adverse reactions of Taeeumin Soeumin Soyangin by Taeeumjowi-tang. Korean $J$ Oriental Physiol Pathol 2008;22:1600-5.

36. Haller CA, Benowitz NL. Adverse cardiovascular and central nervous system events associated with dietary supplements containing ephedra alkaloids. $N$ Engl J Med 2000;343:1833-8.

37. Chen S, Lv F, Gao J, Lin J, Liu Z, Fu Y, et al. HLA class II polymorphisms associated with the physiologic characteristics defined by traditional Chinese medicine: linking modern genetics with an ancient medicine. $J$ Altern Complement Med 2007;13:231-9.

38. Hankey A. The scientific value of Ayurveda. J Altern Complement Med 2005;11:221-5.

39. Zwickey H, Schiffke HC. Genetic correlates of Chinese medicine: in search of a common language. $J$ Altern Complement Med 2007;13:183-4.

40. Won HH, Jang E, Kim KK, Park YK, Kim YJ, Kim YS, et al. A genome-wide scan for the Sasang constitution in a large Korean family suggests significant linkage at chromosomes 8q11.22-23 and 11q22.1-3. J Altern Complement Med 2009, in press.

Received March 12, 2009; accepted May 22, 2009 


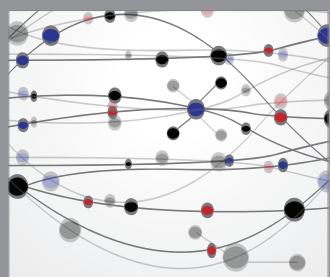

The Scientific World Journal
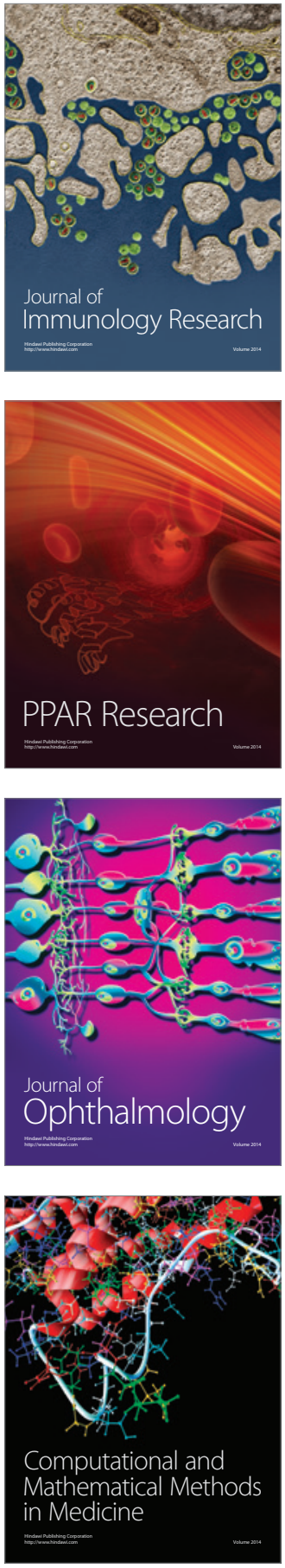

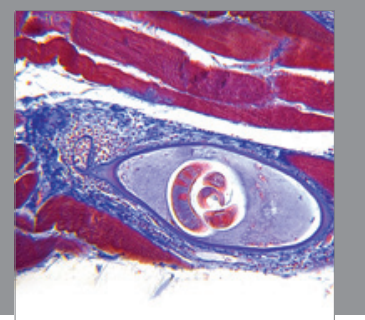

Gastroenterology

Research and Practice
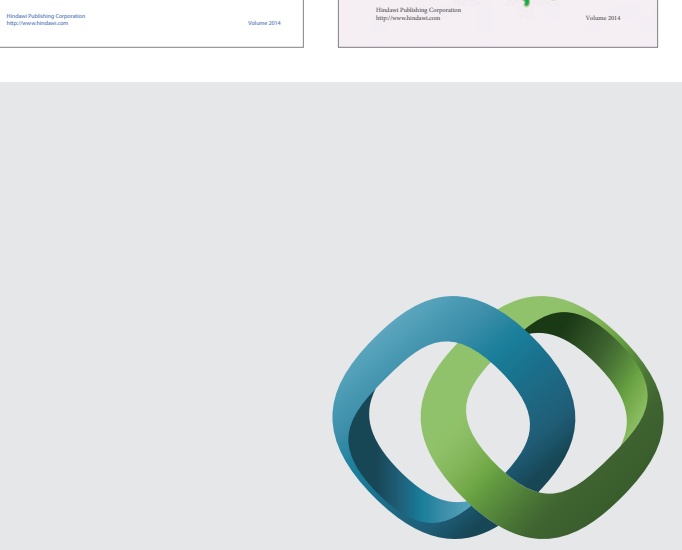

\section{Hindawi}

Submit your manuscripts at

http://www.hindawi.com
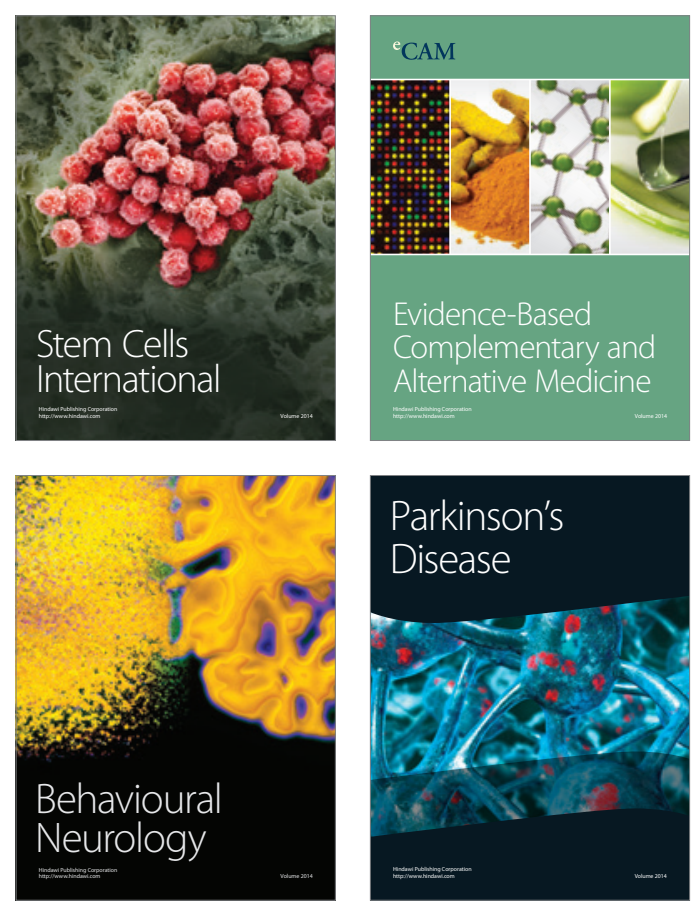

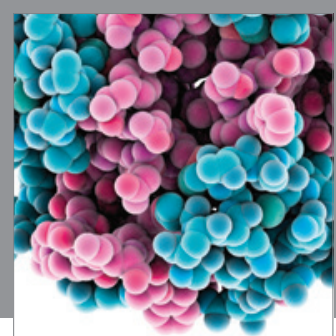

Journal of
Diabetes Research

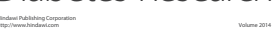

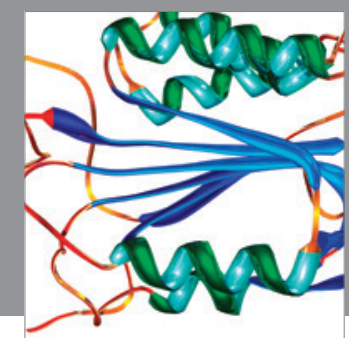

Disease Markers
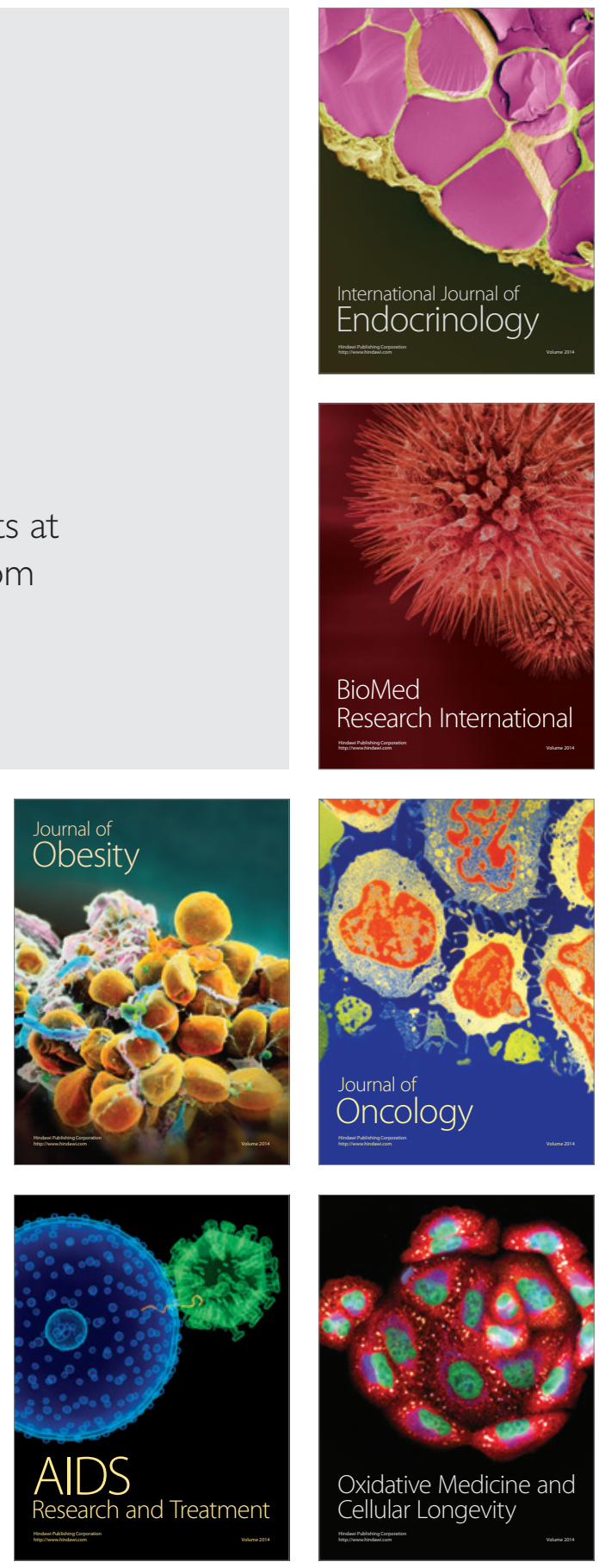